\title{
QUANTITATIVE ANALYSIS OF COMPETITION MODELS
}

\author{
CRISTINA CHIRALT, ANTONI FERRAGUT, ARMENGOL GASULL, PURA VINDEL
}

\begin{abstract}
We study a 2-species Lotka-Volterra type differential system, modeling competition between two species and having a coexistence equilibrium in the first quadrant. In case that this equilibrium is of saddle type, its stable manifold divides the first quadrant into two zones. Then, depending on the zone where the initial condition lies, one of the species will extinct and the other will go to an equilibrium. Using this separatrix we introduce a measure to discern which species has more chance of surviving. This measure is given by a non-negative real number $\kappa$, that we will call persistence ratio, that only depends on the parameters of the system. In some cases, we can give simple explicit expressions for $\kappa$. When this is not possible, we use several dynamical tools to obtain effective approximations of it.
\end{abstract}

\section{INTRODUCTION}

In competition models two or more species struggle for a limited source, like food or territory. Following Murray [15, Sec. 3.5], in this work we consider a simple 2-species Lotka-Volterra competition model for which each species has logistic growth in absence of the other. More specifically, we consider the quadratic differential system

$$
\begin{aligned}
& \dot{x}=\frac{d x}{d t}=x\left(\lambda-\alpha_{1} x-\alpha_{2} y\right), \\
& \dot{y}=\frac{d y}{d t}=y\left(\mu-\beta_{1} x-\beta_{2} y\right),
\end{aligned}
$$

where $\alpha_{1}, \alpha_{2}, \beta_{1}, \beta_{2}, \lambda$ and $\mu$ are positive parameters and $t \in \mathbb{R}$ is the time. For short we name $\mathcal{X}$ and $\mathcal{Y}$ the species with respective populations $x$ and $y$.

We will study the case when the above system has an equilibrium $\mathbf{p}$ in the open first quadrant which is of saddle type. Recall that this situation allows to show for this 2-species model the so called Principle of competitive exclusion: when two species compete for the same limited resources, one of them usually becomes extinct. System (1) is known to be a good model for several kinds of species in competition, as shown in the classical works of Gause and Leslie, see [10, 12].

To the best of our knowledge, there is not a quantitative version of this principle. The aim of this paper is to fill this gap. We will introduce a non-negative real (even infinity) number $\kappa$, that we will call persistence ratio, that will measure which of both species has more chance of surviving and that depends only on the parameters of the model. As we will see, the computation of this number relays on the knowledge of the expression of the stable manifold of $\mathbf{p}, \mathcal{S}=\mathcal{W}^{s}(\mathbf{p})$, where $\mathbf{p}$ is a hyperbolic saddle in the open first quadrant. Since for the most of the cases this manifold is not algebraic, this computation is not easy. In this work we will approach $\kappa$ by looking for algebraic approximations of $\mathcal{S}$. These approximations will be obtained following similar tools to the ones developed in $[7,8,9]$. For fixed values of the parameters of the model, an alternative approach for obtaining $\kappa$ would be to apply numerical methods to compute $\mathcal{S}$. However, in this work, we center our efforts in obtaining analytic results.

Next we introduce $\kappa$ for system (1), in the case that it has a saddle point in the first open quadrant. In fact, in this situation it can be seen that the system has two more

2010 Mathematics Subject Classification. 34A05,34A34,34C05.

Key words and phrases. Lotka-Volterra differential system, invariant algebraic curve, separatrix, algebraic approximation. 


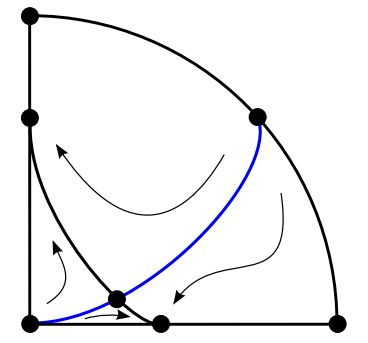

(a) $1 \leq \frac{\mu}{\lambda}<\min \left\{\frac{\alpha_{2}}{\beta_{2}}, \frac{\beta_{1}}{\alpha_{1}}\right\}$

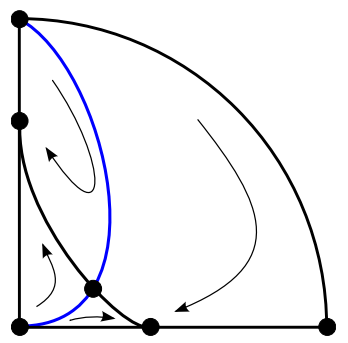

(b) $\frac{\alpha_{2}}{\beta_{2}} \leq 1<\frac{\mu}{\lambda}<\frac{\beta_{1}}{\alpha_{1}}$

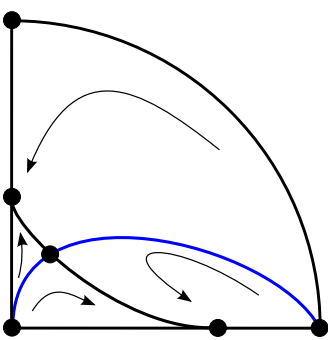

(c) $\frac{\mu}{\lambda}<\frac{\beta_{1}}{\alpha_{1}} \leq 1<\frac{\alpha_{2}}{\beta_{2}}$

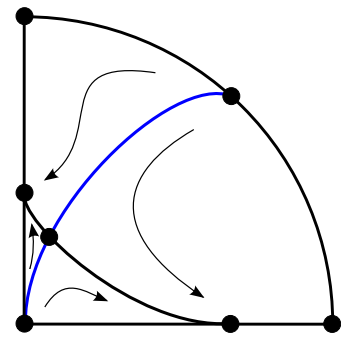

(d) $\frac{\mu}{\lambda}<1<\min \left\{\frac{\alpha_{2}}{\beta_{2}}, \frac{\beta_{1}}{\alpha_{1}}\right\}$

Figure 1. Phase portraits in the first quadrant of the Poincaré sphere of system (1) having a saddle in the open first quadrant. The separatrix $\mathcal{S}$ of the saddle is presented in blue. Note that either (a) and (d), and (b) and (c), are equivalent after swapping variables.

equilibria on the positive axes $\left(0, \mu / \beta_{2}\right)$ and $\left(\lambda / \alpha_{1}, 0\right)$ that correspond to extinction of the first or the second species, respectively. The different phase portraits of system (1) having a saddle in the first quadrant of the Poincare disc are given in Figure 1, where the arc of circle corresponds to the points at infinity. See $[1,11,19]$ for more information about the Poincaré compactification. In fact, phase portraits and integrability of general quadratic Lotka-Volterra systems have been studied in many works, see for instance $[2,16,17]$ and the references therein.

Given any positive real number $R>0$, consider the two areas

$$
\begin{aligned}
& A^{+}(R)=\mu_{\mathcal{L}}\left(\left\{z_{0} \in[0, R]^{2}: \omega\left(\gamma_{z_{0}}\right)=\left(0, \mu / \beta_{2}\right)\right\}\right), \\
& A^{-}(R)=\mu_{\mathcal{L}}\left(\left\{z_{0} \in[0, R]^{2}: \omega\left(\gamma_{z_{0}}\right)=\left(\lambda / \alpha_{1}, 0\right)\right\}\right),
\end{aligned}
$$

where $\mu_{\mathcal{L}}$ is the Lebesgue measure, $\gamma_{z_{0}}$ is the orbit with initial condition $z_{0}=\left(x_{0}, y_{0}\right)$ and $\omega\left(\gamma_{z_{0}}\right)$ is its $\omega$-limit set. The persistence ratio of $\mathcal{Y}$ with respect to $\mathcal{X}$ is

$$
\kappa_{[\mathcal{Y}: \mathcal{X}]}=\lim _{R \rightarrow \infty} \kappa_{[\mathcal{Y}: \mathcal{X}]}(R), \quad \text { where } \quad \kappa_{[\mathcal{Y}: \mathcal{X}]}(R)=\frac{A^{+}(R)}{A^{-}(R)}
$$

Note that we can also define $\kappa_{[\mathcal{X}: \mathcal{Y}]}=1 / \kappa_{[\mathcal{Y}: \mathcal{X}]}$. We will see that the above limit always exists, including infinity. Indeed when it is infinity it means that $\kappa_{[\mathcal{X}: \mathcal{Y}]}=0$. Note that it is non-negative because it comes from a quotient of areas. When there is no confusion we will simply write $\kappa=\kappa_{[\mathcal{Y}: \mathcal{X}]}$ or $\kappa(R)=\kappa_{[\mathcal{Y}: \mathcal{X}]}(R)$.

If $\kappa_{[\mathcal{Y}: \mathcal{X}]}>1$, then the "measure" of initial populations whose $\omega$-limit is $\left(0, \mu / \beta_{2}\right)$ is bigger that the one for which the $\omega$-limit is $\left(\lambda / \alpha_{1}, 0\right)$. Thus species $\mathcal{Y}$ has more chances of surviving than species $\mathcal{X}$. If $\kappa_{[\mathcal{Y}: \mathcal{X}]}<1$ then the converse happens.

Theorem 1. Consider the differential system (1). It has a saddle in the interior of the first quadrant if and only if

$$
\frac{\alpha_{1}}{\beta_{1}}<\frac{\lambda}{\mu}<\frac{\alpha_{2}}{\beta_{2}}
$$


Moreover, in this situation the persistence ratio of $\mathcal{Y}$ with respect to $\mathcal{X}$ is:

$$
\kappa_{[\mathcal{Y}: \mathcal{X}]}=\left\{\begin{array}{ccc}
0 & \text { when } & \frac{\lambda}{\mu}<\frac{\alpha_{2}}{\beta_{2}} \leq 1, \\
\frac{\alpha_{1}\left(\alpha_{2}-\beta_{2}\right)}{2 \beta_{2}\left(\beta_{1}-\alpha_{1}\right)-\alpha_{1}\left(\alpha_{2}-\beta_{2}\right)}<1 & \text { when } & 1<\frac{\alpha_{2}}{\beta_{2}}<\frac{\beta_{1}}{\alpha_{1}}, \\
1 & \text { when } & 1<\frac{\alpha_{2}}{\beta_{2}}=\frac{\beta_{1}}{\alpha_{1}}, \\
\frac{2 \alpha_{1}\left(\alpha_{2}-\beta_{2}\right)-\beta_{2}\left(\beta_{1}-\alpha_{1}\right)}{\beta_{2}\left(\beta_{1}-\alpha_{1}\right)}>1 & \text { when } \quad 1<\frac{\beta_{1}}{\alpha_{1}}<\frac{\alpha_{2}}{\beta_{2}}, \\
\infty & \text { when } \quad \frac{\mu}{\lambda}<\frac{\beta_{1}}{\alpha_{1}} \leq 1 .
\end{array}\right.
$$

Remark 1. The value $\kappa=0$ corresponds to Figure $1(b) ; \kappa=\infty$ corresponds to Figure $1(c)$; when $\kappa \in \mathbb{R}^{+}$we are either in Figure $1(a)$ or $1(d)$, depending on the value of $\lambda / \mu$.

After this theorem, the relation between the ratio of the interspecific and intraspecific competition taxes of each species, that is $\beta_{1} / \alpha_{1}$ for $\mathcal{X}$ and $\alpha_{2} / \beta_{2}$ for $\mathcal{Y}$, determines which one has more chances of surviving: when the ratio of $\mathcal{Y}$ is bigger than the ratio of $\mathcal{X}$, then $\mathcal{Y}$ has more chances than $\mathcal{X}$ of surviving. This statement is coherent with the biological interpretation of the taxes $\alpha_{j}$ and $\beta_{j}, j=1,2$, and shows again that the quadratic LotkaVolterra model is useful to capture and quantify the behavior of two species in competition.

Theorem 1 will be proved in Section 3 (see also Section 2.4). As we will see its proof does not need to know the exact expression of $\mathcal{S}$, but only its asymptotic behavior at infinity, that is contained in the singularities of (1) at infinity. We will prove our result using a simplified version of system (1), obtained by an adimensionalization procedure, see system (2) and Theorem 5 in Section 2. We remark that our reparametrization of $x, y$ and $t$ is slightly different to the one used in [15, Sec. 3.5].

It is also natural to suppose that the total number of individuals $x$ and $y$ is bounded by a constant, say $R>0$, due to some ecological restrictions. In this situation, the relevant ratio is no more $\kappa$ but $\kappa(R)$. When $\mathcal{S}$ is contained into an algebraic curve (see Theorem 2 below), we can exactly compute the ratio $\kappa(R)$. From the results of Seidenberg [18, Thm 8], see also [4, Thm 13], it is not difficult to prove that when $\mu / \lambda \in \mathbb{R}^{+} \backslash \mathbb{Q}$ then all the curves through the origin except the axes are non-analytic. In particular $\mathcal{S}$ is not contained into an invariant algebraic curve. Hence, unfortunately in the most of the cases $\mathcal{S}$ is not algebraic. In fact, based on the results of Moulin-Ollagnier [14], we can characterize all the cases when $\mathcal{S}$ is algebraic.

Theorem 2. The families of systems (1) with $\mu \geq \lambda$ having a saddle in the first quadrant whose stable manifold $\mathcal{S}$ is contained into an invariant algebraic curve $f(x, y)=0$ of degree $N$ satisfy one of the following sets of conditions:

(i) $\mu=\lambda, \alpha_{1}-\beta_{1}<0, \alpha_{2}-\beta_{2}>0, N=1$ and

$$
f(x, y)=\left(\alpha_{1}-\beta_{1}\right) x+\left(\alpha_{2}-\beta_{2}\right) y .
$$

(ii) $\mu=2 \lambda, \beta_{1}=\left(2 \alpha_{1} \alpha_{2}-3 \alpha_{1} \beta_{2}\right) /\left(\alpha_{2}-2 \beta_{2}\right), \alpha_{2}>2 \beta_{2}, N=2$ and

$$
f(x, y)=y-\frac{\beta_{2}}{2 \lambda}\left(\frac{\alpha_{1} x}{\alpha_{2}-2 \beta_{2}}-y\right)^{2} .
$$

(iii) $\mu=3 \lambda, \alpha_{2}=7 \beta_{2} / 3, \beta_{1}=5 \alpha_{1}, N=3$ and

$$
f(x, y)=y+\frac{2 y\left(3 \alpha_{1} x-\beta_{2} y\right)}{3 \lambda}-\frac{\left(3 \alpha_{1} x-\beta_{2} y\right)^{3}}{9 \beta_{2} \lambda^{2}} .
$$


(iv) $\mu=4 \lambda, \alpha_{2}=9 \beta_{2} / 4, \beta_{1}=6 \alpha_{1}, N=4$ and

$$
f(x, y)=y+\frac{y\left(8 \alpha_{1} x-3 \beta_{2} y\right)}{4 \lambda}+\frac{3 y\left(4 \alpha_{1} x-\beta_{2} y\right)^{2}}{16 \lambda^{2}}-\frac{\left(4 \alpha_{1} x-\beta_{2} y\right)^{4}}{64 \beta_{2} \lambda^{3}} .
$$

(v) $\mu=3 \lambda / 2, \alpha_{2}=8 \beta_{2} / 3, \beta_{1}=7 \alpha_{1} / 2, N=4$ and

$$
f(x, y)=y^{2}-\frac{\left(3 \alpha_{1} x-2 \beta_{2} y\right)^{2}\left(3 \alpha_{1} x+4 \beta_{2} y\right)}{12 \beta_{2}^{2} \lambda}+\frac{\left(3 \alpha_{1} x-2 \beta_{2} y\right)^{4}}{36 \beta_{2}^{2} \lambda^{2}} .
$$

(vi) $\mu=6 \lambda, \alpha_{2}=13 \beta_{2} / 6, \beta_{1}=8 \alpha_{1}, N=6$ and

$$
\begin{aligned}
f(x, y) & =y+\frac{y\left(12 \alpha_{1} x-5 \beta_{2} y\right)}{6 \lambda}+\frac{y\left(54 \alpha_{1}^{2} x^{2}-36 \alpha_{1} \beta_{2} x y+5 \beta_{2}^{2} y^{2}\right)}{18 \lambda^{2}} \\
& +\frac{y\left(12 \alpha_{1} x-5 \beta_{2} y\right)\left(6 \alpha_{1} x-\beta_{2} y\right)^{2}}{108 \lambda^{3}}+\frac{5 y\left(6 \alpha_{1} x-\beta_{2} y\right)^{4}}{1296 \lambda^{4}}-\frac{\left(6 \alpha_{1} x-\beta_{2} y\right)^{6}}{7776 \beta_{2} \lambda^{5}} .
\end{aligned}
$$

Moreover:

(1) The families (i) and (ii) are Liouville integrable.

(2) The families (iii) to (vi) are rationally integrable.

The families not satisfying any of the above sets of conditions have a non-algebraic stable manifold $\mathcal{S}$.

Remark 2. In Theorem 2 we only study the case $\mu \geq \lambda$ because the cases $\mu\langle\lambda$ and $\mu\rangle \lambda$ are equivalent, see Remark 3 below.

Section 4 is devoted to prove Theorem 2. In Section 5 we compute $\kappa$ and $\kappa(R)$ for some of the cases of Theorem 2, that is when $\mathcal{S}$ is algebraic, see Figures 4 and 5 .

As we conclude from Theorem 2, in the most of the cases it is necessary to obtain algebraic approximations of $\mathcal{S}$, bounding it above and below to approach $\kappa(R)$. Then we can compute the corresponding areas for both approximations. We call them $A_{L}^{+}(R), A_{L}^{-}(R)$ for the lower bound; and $A_{U}^{+}(R), A_{U}^{-}(R)$ for the upper bound. We have

$$
\frac{A_{U}^{+}(R)}{A_{U}^{-}(R)}<\kappa(R)=\frac{A^{+}(R)}{A^{-}(R)}<\frac{A_{L}^{+}(R)}{A_{L}^{-}(R)} .
$$

This may allow us to know whether $\kappa(R)$ is greater or lower than one, see Sections 6 and 7 for specific examples. In fact, these sections are devoted to give algebraic bounds (above and below) of the separatrix $\mathcal{S}$.

In Section 6 we consider an example where all the parameters of the modified model mentioned above (see system (2)) have fixed values. In Section 7 all these new parameters but one are fixed. More concretely, in Section 6 we give a way to compute Padé-type approximations of $\mathcal{S}$ of the form $y=R^{n}(x)$, being $R^{n}(x)$ a rational function of degree $n$ in the numerator and degree $n-1$ in the denominator. As much as $n$ increases, the approximation of $\mathcal{S}$ is better. We study a specific sequence of rational functions that approximate and bound $\mathcal{S}$, for several values of $n$, and we compute and draw a bound of the error. Finally we get an estimation of $\kappa(R)$.

Finally, concerning Section 7, we succeed in computing a bound of $\mathcal{S}$ given by a rational function such as in Section 6. The other bound is given by the quadratic function $f(x, y)=0$ of Theorem 2(ii). An estimation of $\kappa(R)$ is also provided.

\section{PRELiminaries}

2.1. Reduction of the parameters. First of all we reduce the number of parameters of system (1). After the change of variables $(x, y) \mapsto\left(\lambda x / \alpha_{1}, \lambda y / \beta_{2}\right)$ and the change of time $d \tau / d t=\lambda$, system (1) becomes

$$
\begin{aligned}
& \dot{x}=P(x, y)=x(1-x-a y), \\
& \dot{y}=Q(x, y)=y(s-b x-y),
\end{aligned}
$$


where $a=\alpha_{2} / \beta_{2}, b=\beta_{1} / \alpha_{1}, s=\mu / \lambda>0$ and the dot represents the derivatives with respect to $\tau$.

Next lemma bounds the range of values of $a, b$ and $s$.

Lemma 3. System (2) has a saddle in the first quadrant if and only if as $>1$ and $b>s$.

Proof. System (2) has four finite singular points: one at the origin, two more on the axes, located at $(0, s)$ and $(1,0)$, and a fourth one. This happens for all values of the parameters. The only finite singular point of system (2) which might be not on the axes is

$$
\left(\frac{a s-1}{a b-1}, \frac{b-s}{a b-1}\right) \text {. }
$$

The product of its eigenvalues is $-(a s-1)(b-s) /(a b-1)$. Hence the conditions for this singular point to be a saddle in the first quadrant are

$$
\frac{a s-1}{a b-1}>0, \quad \frac{b-s}{a b-1}>0, \quad-\frac{(a s-1)(b-s)}{a b-1}<0,
$$

and that the eigenvalues are real. This happens if and only if $a s>1$ and $b>s$. Hence the lemma follows.

Remark 3. After the change of variables and time $(x, y) \mapsto(y / s, x / s), d \tau / d w=s$, system (2) becomes

$$
\begin{aligned}
& \dot{x}=x(1-x-b y), \\
& \dot{y}=y(1 / s-a x-y),
\end{aligned}
$$

where the dot means now derivative with respect to $w$. Hence it is equivalent to study the case $0<s<1$ and the case $s>1$.

After this remark, we notice that we shall mainly work with the case $s \geq 1$. In this case, and after Lemma 3 , and since $b>s$, in this case we shall have $b>1$.

2.2. Singular points of the differential system (2). We recall that we are interested in the case that system (2) has a saddle point in the open first quadrant. It is not difficult to see that, in this situation, it has three more finite singular points, three nodes located on the axes: one at the origin (a repeller), one on the $x$-axis (an attractor) and another one on the $y$-axis (another attractor).

Moreover, system (2) has three singular points at infinity. They are located in the directions $x=0, y=0$ and $(b-1) x-(a-1) y=0$, because the characteristic polynomial of system (2) at infinity is $x y((b-1) x-(a-1) y)$, see $[1,11,19]$. Depending on the sign of $a-1$ and $b-1$ we have different configurations at infinity, because the position of the third singular point varies with $a$ and $b$ and changes the configuration of the infinite singular points.

The following lemma studies the singular points at infinity of system (2) (see again [1, $11,19]$ for the definition), which allows us to study the behavior at infinity of a polynomial differential system, see Figure 1 . We study only the case $s \geq 1$. The case $s<1$ may follow afterwards easily after Remark 3 .

Lemma 4. System (2) with $s \geq 1$ has three singular points at infinity:

(i) A first singular point lays in the direction $y=0$. It is a saddle.

(ii) A second singular point at infinity lays in the direction $(b-1) x-(a-1) y=0$. If $a>1$ then it is a node. If $a<1$ then it is a saddle. If $a=1$ then it is in the direction $x=0$ and is a saddle-node.

(iii) There is another singular point, located in the direction $x=0$. It is a saddle for $a>1$, a node for $a<1$ and a saddle-node for $a=1$. 
Proof. We deal with the case $a=1$ at the end of the proof. Next we assume that $a \neq 1$. After the change of variables $(u, v)=(y / x, 1 / x)$, that places the origin at the infinite singular point located in the direction $y=0$, system (2) becomes

$$
\dot{u}=u(1-b+(a-1) u+(s-1) v), \quad \dot{v}=v(1+a u-v) .
$$

On $v=0$ (the line at infinity) we have two singular points, say $u=0$ and $u=(b-1) /(a-1)$. The eigenvalues of the first one are 1 and $1-b<0$, hence it is a saddle. For the second one we have the eigenvalues $(a b-1) /(a-1)$ and $b-1>0$, so its behavior depends only on the sign of $a-1$, because $a b-1>a s-1>0$.

It remains to check the infinite singular point in the direction of $x=0$, which cannot be studied with the previous change of variables. So we use the change of variables $(u, v)=$ $(x / y, 1 / y)$, that places the origin at the infinite singular point located in the direction $x=0$. We get the system

$$
\dot{u}=u(1-a+(b-1) u-(s-1) v), \quad \dot{v}=v(1+b u-s v) .
$$

The eigenvalues at the origin are 1 and $1-a$, hence its behavior depends only on the sign of $a-1$.

If $a=1$ then there are two singular points: one in the direction $y=0$, studied before, and another one on the direction $x=0$, with multiplicity two. It is easy to check that it is a saddle-node. The behavior near this point in the first quadrant is the same as the behavior near a node.

In short, $\mathcal{S}$ is the separatrix of the saddle in the first quadrant corresponding to its stable manifold. It joins the origin, the saddle and the corresponding singular point at infinity. The other separatrix (the unstable manifold) joins the saddle with the two finite attractor nodes of the system that are on the axes and is not of our interest here. See Figure 1.

2.3. Invariant algebraic curves. An algebraic curve $f=0$ is invariant by a polynomial differential system $\dot{x}=P(x, y), \dot{y}=Q(x, y)$ if there exists a polynomial $k \in \mathbb{C}[x, y]$, called the cofactor of $f$, such that

$$
(P, Q) \cdot \nabla f=P \frac{\partial f}{\partial x}+Q \frac{\partial f}{\partial y}=k f .
$$

We note that the degree of $k$ is lower than $\max \{\operatorname{deg} P, \operatorname{deg} Q\}$. For the differential system (2), equation (3) writes as

$$
x(1-x-a y) \frac{\partial f}{\partial x}+y(s-b x-y) \frac{\partial f}{\partial y}=\left(k_{0}+k_{1} x+k_{2} y\right) f,
$$

where $k(x, y)=k_{0}+k_{1} x+k_{2} y$ is the cofactor of $f=0$. See [13] for more information about this subject. Theorem 6 below provides all the invariant algebraic curves for the differential system (2) that contain $\mathcal{S}$.

2.4. Equivalent statements of Theorem 1 and Theorem 2. It is not difficult to see that the equivalent versions of Theorems 1 and Theorem 2 for system (2) are the next two results. We only need to use that $a=\alpha_{2} / \beta_{2}, b=\beta_{1} / \alpha_{1}, s=\mu / \lambda$. Note that $a$ and $b$ are the ratio between the interspecific and the intraspecific competition taxes introduced after Theorem 1 and $s$ is the quotient of the eigenvalues at the origin, hence it is natural to work with them. After proving these two theorems, Theorems 1 and Theorem 2 stated in the introduction follow. The proof of Theorem 5 is given in Section 3. The proof of Theorem 6 is deferred to Section 4.

Theorem 5. System (2) has a saddle in the interior of the first quadrant if and only if as $>1$ and $b>s$. Moreover, in this situation the persistence ratio of $\mathcal{Y}$ with respect to $\mathcal{X}$ 
is:

$$
\kappa_{[\mathcal{Y}: \mathcal{X}]}=\left\{\begin{array}{cc}
0 & \text { when } \frac{1}{s}<a \leq 1, \\
\frac{a-1}{2(b-1)-(a-1)}<1 & \text { when } 1<a<b, \\
1, & \text { when } 1<a=b, \\
\frac{2(a-1)-(b-1)}{b-1}>1 & \text { when } 1<b<a \\
\infty & \text { when } s<b \leq 1 .
\end{array}\right.
$$

Theorem 6. The families of systems (2) with $s \geq 1$ having a saddle in the first quadrant whose stable separatrix $\mathcal{S}$ is contained into an invariant algebraic curve of degree $N$, $f(x, y)=0$, satisfy one of the following sets of conditions:

(i) $s=1, a>1, b>1, N=1$ and

$$
f(x, y)=(b-1) x-(a-1) y .
$$

(ii) $s=2, b=(2 a-3) /(a-2), a>2, N=2$ and

$$
f(x, y)=y-\frac{1}{2}\left(\frac{x}{a-2}-y\right)^{2} \text {. }
$$

(iii) $s=3, a=7 / 3, b=5, N=3$ and

$$
f(x, y)=y+\frac{2}{3}(3 x-y) y-\frac{1}{9}(3 x-y)^{3} .
$$

(iv) $s=4, a=9 / 4, b=6, N=4$ and

$$
f(x, y)=y+\frac{1}{4}(8 x-3 y) y+\frac{3}{16}(4 x-y)^{2} y-\frac{1}{64}(4 x-y)^{4} .
$$

(v) $s=3 / 2, a=8 / 3, b=7 / 2, N=4$ and

$$
f(x, y)=y^{2}-\frac{1}{12}(3 x-2 y)^{2}(3 x+4 y)+\frac{1}{36}(3 x-2 y)^{4} .
$$

(vi) $s=6, a=13 / 6, b=8, N=6$ and

$$
\begin{aligned}
f(x, y) & =y+\frac{1}{6}(12 x-5 y) y+\frac{1}{18}\left(54 x^{2}-36 x y+5 y^{2}\right) y \\
& +\frac{1}{108}(12 x-5 y)(6 x-y)^{2} y+\frac{5}{1296}(6 x-y)^{4} y-\frac{1}{7776}(6 x-y)^{6} .
\end{aligned}
$$

Moreover:

(1) The families (i) and (ii) are Liouville integrable.

(2) The families (iii) to (vi) are rationally integrable.

The families not satisfying any of the above sets of conditions have a non-algebraic stable manifold $\mathcal{S}$.

Notice that the cases $s<1$ for which the separatrix $\mathcal{S}$ is algebraic can be studied similarly by using Remark 3. Figure 2 illustrates the algebraic curves of Theorem 6 .

\section{Proof of Theorem 5}

The first part of the theorem follows after Lemma 3. We prove the second part in the case $a, b>1$, the other cases may follow using the same arguments. We remind that system (2) has an infinite singular point in the first quadrant in the direction $(b-1) x-(a-1) y=0$, which is where the manifold $\mathcal{S}$ passes through. This means that

$$
\lim _{x \rightarrow \infty} \frac{y(x)}{x}=m
$$




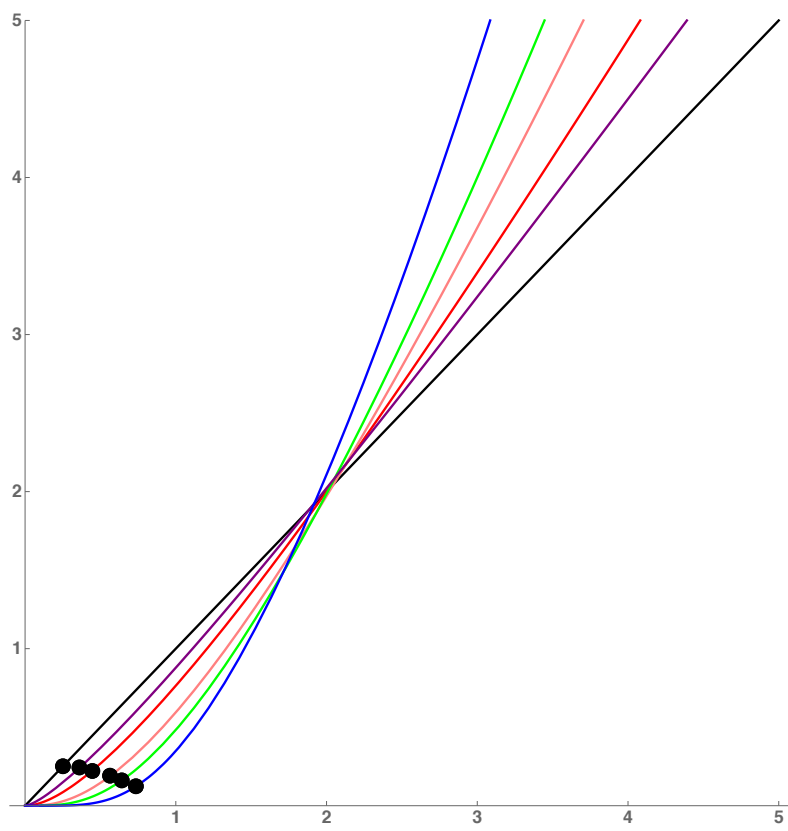

FiguRE 2. Graph of the separatrices $\mathcal{S}$ and the saddle for all families (i)-(vi) altogether (resp. black, red, pink, green, purple, blue). For family (i) we have set $a=b=3$. For family (ii) we have set $a=5 / 2$.

where $y(x)$ represents here $\mathcal{S}$ and $m=(b-1) /(a-1)>0$. Moreover we have by the l'Hôpital rule that

$$
\lim _{x \rightarrow \infty} y^{\prime}(x)=m
$$

which means that $y(x)$ is invertible for $x$ big enough.

If $m \leq 1$, that is $1<b \leq a$, then

$$
\kappa=\lim _{R \rightarrow \infty} \frac{A^{+}(R)}{A^{-}(R)}=\lim _{R \rightarrow \infty} \frac{R^{2}-\int_{0}^{R} y(x) d x}{\int_{0}^{R} y(x) d x}=\lim _{R \rightarrow \infty} \frac{R^{2}}{\int_{0}^{R} y(x) d x}-1 .
$$

Again using the l'Hôpital rule, we get

$$
\kappa=\lim _{R \rightarrow \infty} \frac{A^{+}(R)}{A^{-}(R)}=\lim _{R \rightarrow \infty} \frac{2 R}{y(R)}-1=\frac{2}{m}-1=\frac{2(a-1)-(b-1)}{b-1} .
$$

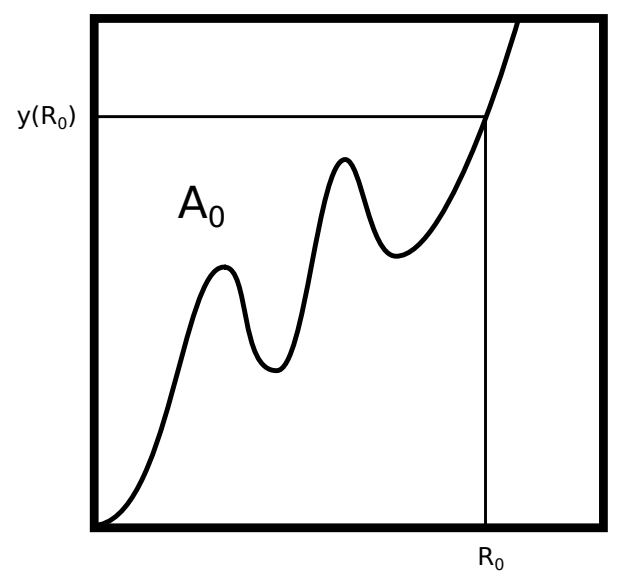

FiguRE 3 . The region $A_{0}$ in the case $m>1$.

If $m \geq 1$, that is $1<a \leq b$, let $R_{0}>0$ be some value for which $y(x)$ is invertible for $x>R_{0}$. Let $A_{0}$ be the area bounded by $\mathcal{S}, y=y\left(R_{0}\right)$ and $x=0$, see Figure 3 . Then 
we can consider $y^{-1}(x)$ instead of $y(x), m^{-1}$ instead of $m$ and $A^{-}(R) / A^{+}(R)$ instead of $A^{+}(R) / A^{-}(R)$ and apply the computations of the case $m<1$. We obtain

$$
\kappa=\lim _{R \rightarrow \infty} \frac{A^{+}(R)}{A^{-}(R)}=\frac{a-1}{2(b-1)-(a-1)} .
$$

In the cases $a \leq 1$ or $b \leq 1$, we can apply similar arguments with $m \in\{0, \infty\}$. Then the theorem follows.

\section{Algebraic separatrices of system (2). Proof of Theorem 6}

Proof of Theorem 6. From [14] (see also [3]) we get that system (2) having a saddle in the first quadrant has no invariant algebraic curves of degree greater than six.

To obtain the invariant algebraic curves of given degree we must deal with the equation (4). When the cofactor is known, this is equivalent to a linear system of equations. The use of standard techniques of the Darboux Theory of Integrability allow us to previously compute the cofactor in terms of the coefficients of the system and the degree of the curve. Then, tedious but straightforward computations show that the only families having an invariant algebraic curve of degree at most six joining the origin and the saddle in the first quadrant (that is, containing $\mathcal{S}$ ) are the ones provided by the theorem.

Concerning integrability, it is well-known that quadratic systems having three invariant algebraic curves have a Darboux inverse integrating factor, $V$, see for instance $[6,13]$ for more information about the inverse integrating factor, respectively and the Darboux Theory of integrability. In particular, system (i) has the inverse integrating factor

$$
V(x, y)=x^{\frac{a}{a-1}} y^{\frac{b}{b-1}}((b-1) x-(a-1) y)^{-\frac{a+b-2}{(a-1)(b-1)}} .
$$

Similarly, system (ii) has the Darboux inverse integrating factor

$$
V(x, y)=x^{2} y^{a-1}\left(\left(\frac{x}{a-2}-y\right)^{2}-2 y\right)^{-\frac{2 a-3}{2}} \text {. }
$$

\begin{tabular}{|c|c|c|}
\hline Family & First integral & Expression of $g$ \\
\hline \hline (ii) $)_{a=5 / 2}$ & $x^{2} y f^{-2} g^{-1}$ & $4-4(2 x+y)+(2 x-y)^{2}$ \\
\hline (iii) & $x^{3} y f^{-2}$ & $16-8(4 x+y)+(4 x-y)^{2}$ \\
\hline (iv) & $x^{4} y f^{-2} g^{-1}$ & 4 \\
\hline (v) & $x^{3} y^{2} g^{-2}$ & $y^{2}+\frac{(3 x-2 y)\left(3 x^{2}+2 x y+4 y^{2}\right)}{4}-\frac{(3 x-2 y)^{3}(x+y)}{6}+\frac{(3 x-2 y)^{5}}{108}$ \\
\hline (vi) & $x^{6} y f^{-2}$ & \\
\hline
\end{tabular}

TABLE 1. Rational first integrals of system (ii) with $a=5 / 2$ and of systems (iii)(vi) of Theorem 6 . In all cases $f=0$ is the corresponding invariant algebraic curve containing $\mathcal{S}$.

In the specific case $a=5 / 2$ system (ii) has a rational first integral, see Table 1 . Indeed Table 1 shows a rational first integral for each one of the systems (iii) to (vi).

Remark 4. The numerator of all the first integrals in Table 1 has the form $x^{p} y^{q}$, for some $p, q \in \mathbb{N}$. See [5] for details about this fact.

Remark 5. We note that there is a subfamily of system (2) found in [14] having invariant algebraic curves of arbitrary degree $2 \ell, \ell \in \mathbb{N}$ (and, in particular, of degree greater than six):

$$
\dot{x}=x\left(1-\frac{x}{2}+y\right), \quad \dot{y}=y\left(-\frac{2 \ell+1}{2 \ell-1}-\frac{x}{2}-y\right), \quad \ell \in \mathbb{N} .
$$


This system is not relevant for our study because it can be seen that it never has a saddle in the first quadrant.

\section{Computation of $\kappa(R)$ When $\mathcal{S}$ is algebraic}

In this section first we compute $\kappa$ and $\kappa(R), R>0$, for system (2) in case (ii) of Theorem 6, when the separatrix $\mathcal{S}$ is contained into the quadratic algebraic curve $\mathcal{F}(x, y)=$ $(x /(a-2)-y)^{2}-2 y=0$. Recall that in this situation we have $s=2, b=(2 a-3) /(a-2)$ and $a>2$.

Let $R>0$ and consider the square $[0, R]^{2}$. We write $\mathcal{S}=\{(x, h(x)): x>0\} \subset\{\mathcal{F}=0\}$, where

$$
h(x)=1+\frac{x}{a-2}-\sqrt{1+\frac{2 x}{a-2}} .
$$

See the blue curve in Figure 9 in Section 7.1, which we call the right branch of $\mathcal{F}=0$. Note that $h^{-1}(y)=(a-2)(y+\sqrt{2 y})$.

If $h(R) \leq R$ then we have $A^{-}(R)=\int_{0}^{R} h(s) d s$ and

$$
\kappa(R)=\frac{A^{+}(R)}{A^{-}(R)}=\frac{R^{2}-\int_{0}^{R} h(s) d s}{\int_{0}^{R} h(s) d s} .
$$

We compute $A^{-}(R)$ :

$$
A^{-}(R)=R+\frac{R^{2}}{2(a-2)}-\frac{a-2}{3}\left(\left(1+\frac{2 R}{a-2}\right)^{3 / 2}-1\right) .
$$

Hence

$$
\kappa(R)=\frac{R^{2}-R-\frac{R^{2}}{2(a-2)}+\frac{a-2}{3}\left(\left(1+\frac{2 R}{a-2}\right)^{3 / 2}-1\right)}{R+\frac{R^{2}}{2(a-2)}-\frac{a-2}{3}\left(\left(1+\frac{2 R}{a-2}\right)^{3 / 2}-1\right)} .
$$

When $R \rightarrow \infty$, we have $\kappa=2 a-5$.

If $h(R)>R$ then $A^{+}(R)=\int_{0}^{R} h^{-1}(s) d s$ and

$$
\kappa(R)=\frac{A^{+}(R)}{A^{-}(R)}=\frac{\int_{0}^{R} h^{-1}(s) d s}{R^{2}-\int_{0}^{R} h^{-1}(s) d s} .
$$

We compute $A^{+}(R)$ :

$$
A^{+}(R)=\frac{a-2}{2}\left(R^{2}+\frac{4 \sqrt{2}}{3} R^{3 / 2}\right) .
$$

Hence,

$$
\kappa(R)=\frac{\frac{a-2}{2}\left(R^{2}+\frac{4 \sqrt{2}}{3} R^{3 / 2}\right)}{R^{2}-\frac{a-2}{2}\left(R^{2}+\frac{4 \sqrt{2}}{3} R^{3 / 2}\right)} .
$$

When $R \rightarrow \infty$, we have $\kappa=\frac{a-2}{4-a}$.

Notice that the curve $\mathcal{F}=0$ intersects the straight line $y=x$ either at the origin and at $\left(R^{\star}(a), R^{\star}(a)\right)$, where

$$
R^{\star}(a)=2\left(\frac{a-2}{a-3}\right)^{2}>0 .
$$

If $a>3$ then we always have $h(R)<R$. If $2<a \leq 3$ we have $h(R) \leq R$ if and only if $R \leq R^{\star}(a)$. See Figure 4 for a detail of $\kappa(R)$ in this case for several values of $R$. 


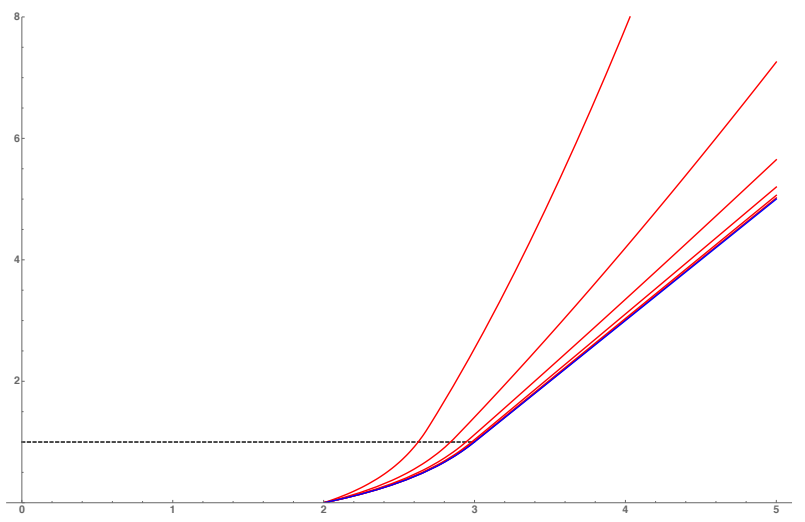

FiguRE 4. Graph of $\kappa(R)$ (red) and $\kappa$ (blue) for case (ii) of Theorem 6 in function of the parameter $a$, for $R=10^{i}, i=1, \ldots, 6$. Notice that at the value of $a$ for which $R=R^{\star}(a)$, the function defining $\kappa(R)$ changes. Moreover, as $R$ increases, $\kappa(R)$ tends to $\kappa$. The dashed horizontal straight line corresponds to height 1 .

Taking $R \rightarrow \infty$, we have $h(R)>R$ for $2<a<3$. Therefore we get

$$
\kappa=\left\{\begin{array}{cl}
\frac{a-2}{4-a}<1, & \text { when } 2<a<3, \\
1, & \text { when } a=3, \\
2 a-5>1, & \text { when } a>3 .
\end{array}\right.
$$

Notice that $\kappa \rightarrow 0$ when $a \rightarrow 2$ and $\kappa \rightarrow \infty$ when $a \rightarrow \infty$. See Figure 4 for a graph of $\kappa$.

Of course these results are coherent with the ones of Theorem 5. In fact, from this theorem, when $b>a$ (which in our situation is equivalent to $2<a<3$, because $b=$ $(2 a-3) /(a-2))$ we get

$$
\kappa=\left.\frac{a-1}{2(b-1)-(a-1)}\right|_{b=(2 a-3) /(a-2)}=\frac{a-2}{4-a} .
$$

Similarly, when $b<a$,

$$
\kappa=\left.\frac{2(a-1)-(b-1)}{b-1}\right|_{b=(2 a-3) /(a-2)}=2 a-5 .
$$

The ratios $\kappa(R)$ and $\kappa$ can also be easily computed using the same arguments for system (2) in all the other cases of Theorem 6 . As an example, Figure 5 shows a numerical plot of $\kappa(R)$, based on the analytic expression of $\mathcal{S}$, for all values of $R<100$, for case (iii) of Theorem 6. Notice that, by Theorem 5, in this case we have $\kappa=\lim _{R \rightarrow \infty} \kappa(R)=1 / 5$.

\section{Algebraic approximation of $\mathcal{S}$ for given values of the parameters}

In this section we show with an example how to approximate and bound (above and below) the invariant separatrix $\mathcal{S}$ by algebraic curves for given values of the parameters $a, b, s$ of system (2). We shall compute Padé-type approximations for bounding the separatrix $\mathcal{S}$.

The Padé approximation is a type of rational approximation of smooth functions $y=$ $F(x)$. Let $F$ be represented by a power series, for example at the origin. Then the rational function $R^{m, n}(x)=\frac{P^{m}(x)}{Q^{n}(x)}$ is said to be the $(m, n)$-Padé approximation of $y=F(x)$ (or, simply, the Padé approximation of $y=F(x))$ if $P^{m}(x)$ is a polynomial of degree $m, Q^{n}(x)$ is a polynomial of degree $n$, and

$$
F(x)-R^{m, n}(x)=O\left(x^{m+n+1}\right) .
$$

Note that given $m$ and $n$ the Padé approximation is unique. 


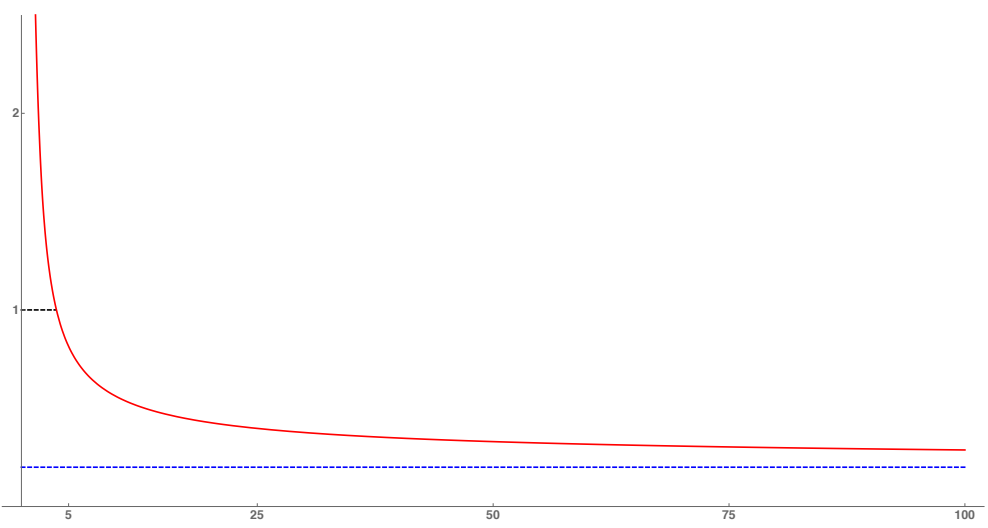

FIguRE 5. Graph of $\kappa(R)$ in case (iii) of Theorem 6. The dashed blue straight line corresponds to $\kappa=1 / 5$, to which the curve $\kappa(R)$ tends as $R \rightarrow \infty$. Notice that $\kappa(R)=1$ for $R \sim 3.7114$.

We shall construct two rational functions of degree $n>2$ in the numerator and $n-1$ in the denominator. They will not be $(n, n-1)$-Padé approximations, strictly speaking, because they will only approximate the Taylor series expansion of $\mathcal{S}$ up to order $2 n-3$ (not $2 n-1$ ). We shall leave three parameters free in order to get upper and lower global approximations.

We explain now how to achieve the expression of the rational functions. We present an algorithm to find them that can be applied to any $n$ :

(1) We consider the rational curve $y=R^{n}(x)=\sum_{i=0}^{n} a_{i} x^{i} / \sum_{i=0}^{n-1} b_{i} x^{i}$, where $a_{i}, b_{i}$ are constants to be determined. We compute its power series expansion at the saddle point. We remark that we want to have a good approximation of $\mathcal{S}$ at the saddle.

(2) We compute the power series expansion $y(x)$ of $\mathcal{S}$ at the saddle from the equality $P(x, y(x)) y^{\prime}(x)-Q(x, y(x))=0$. Of course this power series and the power series of $R^{n}(x)$ must be equal up to some order. Equaling the coefficients of both power series, we can obtain all the $a_{i}$ and also $b_{0}, \ldots, b_{n-4}$.

We notice that we assume the denominator of $R^{n}(x)$ to have one degree less than its numerator, because we want $R^{n}(x)$ to be asymptotic to a straight line at infinity. Recall that $\mathcal{S}$ is asymptotic to a straight line at infinity because its $\omega$-limit is the singular point at infinity in the first quadrant.

(3) The value of $b_{n-3}$ is computed from the equation $a_{n} / b_{n-1}=\gamma$, with $\gamma \in\{(c+$ $1) / c, c /(c+1)\}$, where $c>0$ is a constant to be determined. This distinction will provide us afterwards the two approximations $y=R_{1}^{n}(x)$ and $y=R_{2}^{n}(x)$, respectively.

(4) After this constraint, we have $\lim _{x \rightarrow \infty} \frac{R^{n}(x)}{x}=\gamma x$.

(5) We define $M_{R_{i}^{n}(x)}=\left[(P, Q) \cdot\left(-\left(R_{i}^{n}\right)^{\prime}(x), 1\right)\right]_{y=R_{i}^{n}(x)}$. The values of $b_{n-2}, b_{n-1}$ and $c$ may be fixed in such a way that $M_{R_{1}^{n}(x)}>0$ and $M_{R_{2}^{n}(x)}<0$ on $x>0$.

(6) Since the gradients $\left(-\left(R_{i}^{n}\right)^{\prime}(x), 1\right)$ of both curves $y=R_{i}^{n}(x)$ point upwards and the signs of $M_{R_{1}^{n}}$ and $M_{R_{2}^{n}}$ are constant and opposite, we know that we have achieved our bounds. Moreover, they both are good approximations of $\mathcal{S}$ when we are close enough to the saddle.

As an example we take $a=b=3$ and $^{1} s=1567 / 807 \approx 1.94$. The saddle point is located at $(649 / 1076,427 / 3228) \approx(0.60,0.13)$ and its eigenvalues are as well rational. We have computed the expressions of $R_{1}^{n}(x)$ and $R_{2}^{n}(x)$ for $n=3,4,5,6,7$. They are plotted in Figure 6. For example, if $n=3$ their expressions are

$$
R_{1}^{3}(x)=\frac{7\left(50269309450649849-386504357665189260 x+1899039830299867248 x^{2}+1102851944451753408 x^{3}\right)}{380904\left(17785828334447+135116522993760 x+13511652299376 x^{2}\right)}
$$

\footnotetext{
${ }^{1}$ This value of $s$ is fixed to avoid square roots during the computation. This idea will be also used in (7).
} 


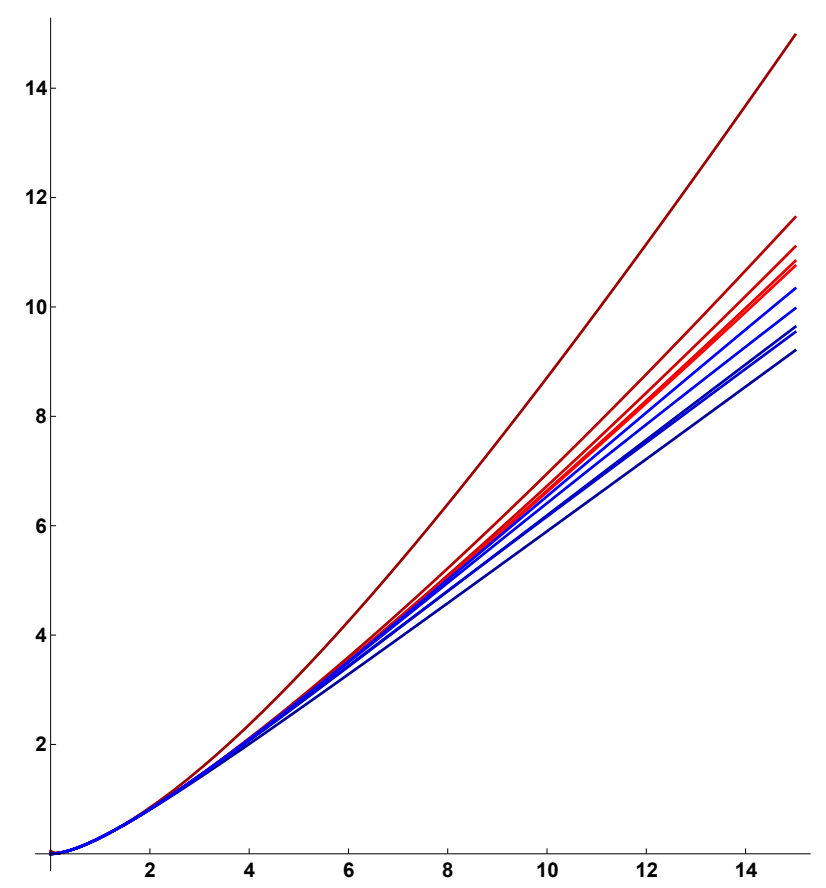

FiguRE 6. Upper (red) and lower (blue) bounds of $\mathcal{S}$ when $a=b=3$ and $s=$ $1567 / 807$ for $n=3,4,5,6,7$. The separatrix lays in between. Note that larger values of $n$ provide better approximations of $\mathcal{S}$.

and

$R_{2}^{3}(x)=\frac{7\left(-545098985687491711+5165387952606894600 x+57820543445825940528 x^{2}+6617111666710520448 x^{3}\right)}{190452\left(4629262639604299+3648146120831520 x+364814612083152 x^{2}\right)}$.

The series expansion of $\mathcal{S}$ at the saddle $(\bar{x}, \bar{y})$ is

$$
\begin{aligned}
y(x)=\frac{427}{3228}+\frac{21}{59}(x-\bar{x})+\frac{643986}{4222453}(x & -\bar{x})^{2}-\frac{30401217673596}{414904527457423}(x-\bar{x})^{3} \\
& +\frac{5885322121483934626350}{131809323613144994382199}(x-\bar{x})^{4}+\mathcal{O}\left((x-\bar{x})^{5}\right) .
\end{aligned}
$$

The corresponding power series of $R_{1}^{3}$ and $R_{2}^{3}$ coincide with this one up to order $2 n-3=3$.

We can compute an upper bound of the relative error $\epsilon_{r}$ for the different values of $n$. This upper bound is computed as:

$$
\left|\frac{R_{1}^{n}-R_{2}^{n}}{R_{2}^{n}}\right|
$$

where $R_{1}^{n}$ is the upper approximation of $\mathcal{S}$ and $R_{2}^{n}$ is its lower approximation, $n>2$. They are plotted in Figure 7. As we can see, the bigger $n$ is, the lower the relative error is. We notice that the relative error is not small for values of $x$ close to zero, this is because the denominator of the relative error is very small (close to zero). For these small values of $x$ one might use a bound of the absolute error, $\left|R_{1}^{n}-R_{2}^{n}\right|$, instead.

Table 2 shows the values $A_{L}^{-}(10)$ and $A_{U}^{-}(10)$ of the areas for the upper and lower bounds of $\mathcal{S}$ for $n=3,4,5,6,7$. They are drawn in Figure 8. From the relations

$$
\frac{A_{U}^{+}(10)}{A_{U}^{-}(10)} \leq \frac{A^{+}(10)}{A^{-}(10)} \leq \frac{A_{L}^{+}(10)}{A_{L}^{-}(10)}
$$

and using the bounds $y=R_{1}^{7}(x)$ and $y=R_{2}^{7}(x)$, we obtain

$$
\kappa(10) \in(2.40919,2.42485) .
$$

Hence in this example there is a bigger chance that the species $\mathcal{X}$ disappears. 


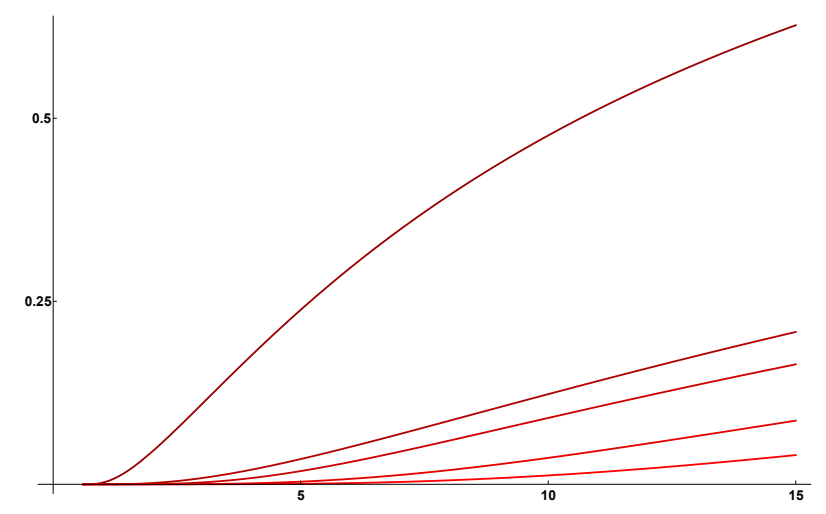

FIGURE 7. An upper bound of the relative error $\epsilon_{r}$ when approximating $\mathcal{S}$, for $a=b=3, s=1567 / 807$ and with $n=3,4,5,6,7$. It is smaller as $n$ increases.

\begin{tabular}{|c|c|c|c|c|c|}
\hline$n$ & 3 & 4 & 5 & 6 & 7 \\
\hline$A_{U}^{-}(10)$ & 36.05 & 30.18 & 29.57 & 29.38 & 29.33 \\
\hline$A_{L}^{-}(10)$ & 27.08 & 28.22 & 28.25 & 28.93 & 29.20 \\
\hline
\end{tabular}

TABLE 2. Areas below the upper and lower bounds of $\mathcal{S}$ for some values of $n$ in a square $[0,10]^{2}$.

FiguRE 8. Areas below the upper and lower bounds of $\mathcal{S}$ for some values of $n$ in a square $[0,10]^{2}$.

\section{Algebraic approximation of $\mathcal{S}$ When a parameter is NOt Fixed}

We can also approximate and bound $\mathcal{S}$ when one of the parameters is not fixed. We study in this section the example with $a=b=3$ fixed and $s>1$ arbitrary. Since $1<s<b$, this means that $s \in(1,3)$. Note that for $s=2$, the separatrix $\mathcal{S}$ is just the algebraic curve given in case (ii) of Theorem 6 , called $\mathcal{F}=0$ in Section 5 . For $s=1$ we are in the situation of item (i) of Theorem 6 and $\mathcal{S}$ is a straight line. When $s=3$ the saddle collapses to the node on the $x$ axis.

So we shall distinguish in the process the cases $1<s<2$ and $2<s<3$. This distinction appears in a natural way, as we shall see later on in Corollary 8.

7.1. First algebraic bound: the algebraic curve $\mathcal{F}=0$. We recall that $\mathcal{F}=0$ is invariant by the flow of system (2) when $s=2, b=(2 a-3) /(a-2)$ and $a>2$, see again case (ii) of Theorem 6 . Solving $\mathcal{F}=0$ with respect to $y$ we have

$$
y=y(x)=1+\frac{x}{a-2} \pm \sqrt{1+\frac{2 x}{a-2}},
$$


for $x \geq 1-a / 2$. Note that although these expressions are not polynomial, of course they provide the same set of points as the algebraic curve $\mathcal{F}=0$. Let

$$
h_{a}(x, y)=y-\left(1+\frac{x}{a-2}-\sqrt{1+\frac{2 x}{a-2}}\right) .
$$

We note that $\mathcal{F}=0$ can be parameterized as

$$
(x, y)=\left(\left(\left(d^{2}-1\right) \delta+2\right) \delta, \frac{\left(d^{2}-1\right)^{2}}{2} \delta^{2}\right),
$$

where $d=\sqrt{\frac{a}{a-2}}>1$. If $\delta>0$ then the parametrization gives a branch of $\mathcal{F}=0$ starting at the origin and, as $\delta$ increases, going to infinity in the first quadrant. We call this branch the right branch of $\mathcal{F}$ at the origin. Note that this right branch is $h_{a}(x, y)=0$ with $x, y>0$ and it is, of course, $\mathcal{S}$. Recall that $h_{a}(x, y)=0$ is the same curve $y=h(x)$ defined in Section 5 .

If $\delta<0$ then we have the branch of $\mathcal{F}=0$ starting at the origin and, as $\delta$ decreases from zero, passing through the second quadrant, crossing the $y$-axis at $\delta=-2 /\left(d^{2}-1\right)$ and going to infinity in the first quadrant. We call this branch the left branch of $\mathcal{F}$. See Figure 9 .

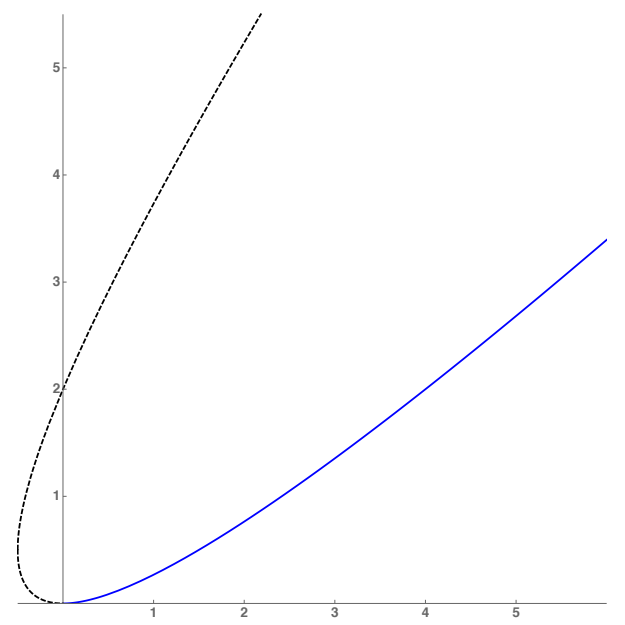

FiguRE 9. The algebraic curve $\mathcal{F}=0$. The right branch, which is of our interest, is presented in blue. It corresponds to $\delta>0$ in the parametrization. The left branch, given by $\delta<0$, is dashed.

When we replace $a$ by its equivalent expression in terms of $d$ into $b=(2 a-3) /(a-2)$ we get $b=\left(d^{2}+3\right) / 2$. The following result holds.

Lemma 7. If either $s \neq 2$ and $b=\left(d^{2}+3\right) / 2$; or $s=2$ and $b \neq\left(d^{2}+3\right) / 2$; or $(s-2)\left(d^{2}+\right.$ $3-2 b)>0$; then the vector field associated to system (2) crosses the right branch of $\mathcal{F}=0$ always in the same direction.

Proof. The relative position of the flow of system (2) with respect to $\mathcal{F}=0$ is controlled by the sign of function

$$
M_{\mathcal{F}}=\left.[(P, Q) \cdot \nabla \mathcal{F}]\right|_{\mathcal{F}=0}=\left.\left[P \mathcal{F}_{x}+Q \mathcal{F}_{y}\right]\right|_{\mathcal{F}=0} .
$$

We next study the sign of $M_{\mathcal{F}}$. We shall prove that it does not vary for all $x, y>0$. Using the parametrization (5), we have

$$
M_{\mathcal{F}}=-\frac{1}{2}\left(d^{2}-1\right)^{2} \delta^{2}\left(\left(d^{2}-1\right) \delta+1\right)\left[2(s-2)+\left(\left(d^{2}-1\right) \delta+2\right) \delta\left(d^{2}+3-2 b\right)\right] .
$$

Only the factor between brackets may change sign, the others have constant sign because $d>1$ and $\delta>0$. Note that this factor can be written as $2(s-2)+\left(d^{2}+3-2 b\right) x$ and 
that $x>0$. If $s=2$ and $d^{2}+3-2 b \neq 0$, then the sign of $M_{\mathcal{F}}$ varies only with the sign of $d^{2}+3-2 b$. If $d^{2}+3-2 b=0$, that is $b=\left(d^{2}+3\right) / 2$, and $s \neq 2$, then the sign of $M_{\mathcal{F}}$ varies only with the sign of $s-2$. Finally if $(s-2)\left(d^{2}+3-2 b\right)>0$ then the expression has constant sign. Then the lemma follows.

Corollary 8. The following statements hold.

(i) If $s=2$ and $b \neq\left(d^{2}+3\right) / 2$, then the stable manifold $\mathcal{S}$ of the saddle is either completely above or completely below the right branch of the parabola $\mathcal{F}=0$. Therefore, we have an algebraic (upper or lower) bound of $\mathcal{S}$.

(ii) If we use the expression $h_{3}(x, y)=y-(1+x-\sqrt{2 x+1})=0$, with $x, y>0$, of the right branch of $\mathcal{F}=0$ in the case $a=b=3$ and $1<s<3$, then we have $M_{h_{3}}=\left.\left[P\left(h_{3}\right)_{x}+Q\left(h_{3}\right)_{y}\right]\right|_{h_{3}=0}=(s-2) y$. Since $y>0$, the function $M_{h_{3}}$ does not change sign for a given value of $s$. Therefore $h_{3}=0$ is a bound of $\mathcal{S}$ which is located either above or below it, depending on whether $s>2$ or $s<2$.

7.2. Second algebraic bound: a Padé approximation. As in the previous section we shall distinguish the cases $s>2$ and $s<2$. Recall that the first bound is given by the right branch of the parabola $\mathcal{F}=0$. To get the other bound we will use again Padé-type approximations of $\mathcal{S}$ at the saddle point.

We shall use the change of parameters

$$
s=\frac{t^{2}-24 t+236}{t^{2}+92}
$$

where $t$ is a new parameter. We note that for any $t \in(2,6)$ we have a unique $s \in(1,2)$ and for any $t \in(-2,2)$ we have a unique $s \in(2,3)$ satisfying the above relation. Moreover, for $t=2$ we have $s=2$. This change of variables will make computations easier, because some square roots will be avoided in the computations.

To obtain the Padé approximations of $\mathcal{S}$ we shall expand this manifold in power series in a neighborhood of the saddle in the first quadrant, which is located at $(\bar{x}, \bar{y})=((3 s-$ 1) $/ 8,(3-s) / 8)$.

We start with the case $1<s<2$. Using (7), we shall take $t \in(2,6)$.

Proposition 9. If $a=b=3$ and $1<s<2$, then the separatrix $\mathcal{S}$ of the saddle point $(\bar{x}, \bar{y})$ in the first quadrant of system (2) is bounded below by $h_{3}(x, y)=y-(x+1-\sqrt{2 x+1})=0$, which is contained into the algebraic curve $\mathcal{F}=0$ with $a=3$, and bounded above by the $(3,2)$-Padé approximation of $\mathcal{S}$ given by

$$
R_{1}=y-\frac{r_{0}(t)-r_{1}(t) x+r_{2}(t) x^{2}+r_{3}(t) x^{3}}{r_{1}(t)+r_{4}(t) x+r_{3}(t) x^{2}}=0
$$

where

$$
\begin{aligned}
r_{0}(t)= & -\left(235447552+2552603648 t-685009152 t^{2}+67418880 t^{3}+10464 t^{4}+33216 t^{5}\right. \\
& \left.-49936 t^{6}+3664 t^{7}-75 t^{8}\right) /\left(4\left(t^{2}+92\right)\right), \\
r_{1}(t)= & 2676800-36990528 t+2300016 t^{2}+117024 t^{3}-900 t^{4}-804 t^{5}+25 t^{6}, \\
r_{2}(t)= & \frac{96\left(t^{2}+92\right)\left(-4195520+10419136 t-758736 t^{2}+41760 t^{3}+4492 t^{4}-580 t^{5}+13 t^{6}\right)}{(t-14)^{2}(t-6)}, \\
r_{3}(t)= & \frac{256\left(t^{2}+92\right)^{2}\left(-7664+2496 t-488 t^{2}-96 t^{3}+7 t^{4}\right)}{(t-14)^{2}(t-6)}, \\
r_{4}(t)= & 4\left(t^{2}+92\right)\left(-494793600-73198656 t-1090144 t^{2}-1271120 t^{3}+190808 t^{4}+4276 t^{5}\right. \\
& \left.-994 t^{6}+25 t^{7}\right) /((t-14)(t-6)(t+2))
\end{aligned}
$$

and $2<t<6$.

Proof. The coordinates of the saddle point in the first quadrant, after changing $s$ by $t$, are:

$$
(\bar{x}, \bar{y})=\left(\frac{(t-14)(t-22)}{4\left(t^{2}+92\right)}, \frac{(t+10)(t+2)}{4\left(t^{2}+92\right)}\right) .
$$


To prove the proposition, we analyze the signs of $M_{R_{1}}$ and $M_{h_{3}}$, see (6). After Corollary 8, we know that the algebraic curve is below the separatrix for $1<s<2$. Indeed, $M_{h_{3}}<0$.

We study now the sign of $M_{R_{1}}$. After long and tedious calculations we obtain:

$$
M_{R_{1}}=\frac{a_{0}(t)+a_{1}(t) x+a_{2}(t) x^{2}+a_{3}(t) x^{3}}{\left(b_{0}(t)+b_{1}(t) x+b_{2}(t) x^{2}\right)^{3}}(x-\bar{x})^{4},
$$

where $a_{i}(t)$ and $b_{i}(t)$ are some polynomials in $t$ with rational coefficients that we do not write. So for $x \neq \bar{x}$ the sign of $M_{R_{1}}$ depends on the signs of these quadratic and cubic polynomials. Using the Sturm algorithm, see [20], we can check that $a_{i}(t)>0$ for all $t \in(2,6), i=0,1,2,3$. Therefore, for $x>0$ the cubic polynomial in the numerator of $M_{R_{1}}$ is positive when $2<t<6$ and $x \neq \bar{x}$.

The same argument shows that $b_{i}(t)>0$ for all $t \in(2,6), i=0,1,2$. So the quadratic polynomial in the denominator of $M_{R_{1}}$ is positive when $\bar{x} \neq x>0$ and $2<t<6$.

Therefore $M_{R_{1}}>0$ in the region $\bar{x} \neq x>0$ and $2<t<6$. Taking additionally into account that both gradients of $h_{3}$ and $R_{1}$ point upwards, the proposition follows.

Figure 10 shows the algebraic bounds of $\mathcal{S}$ for some values of $t$. Note that when $t$ tends to 6 the upper bound tends to a straight line. Indeed, $t=6$ implies $s=1$, for which $\mathcal{S}$ is a straight line, see item (i) in Theorem 6.

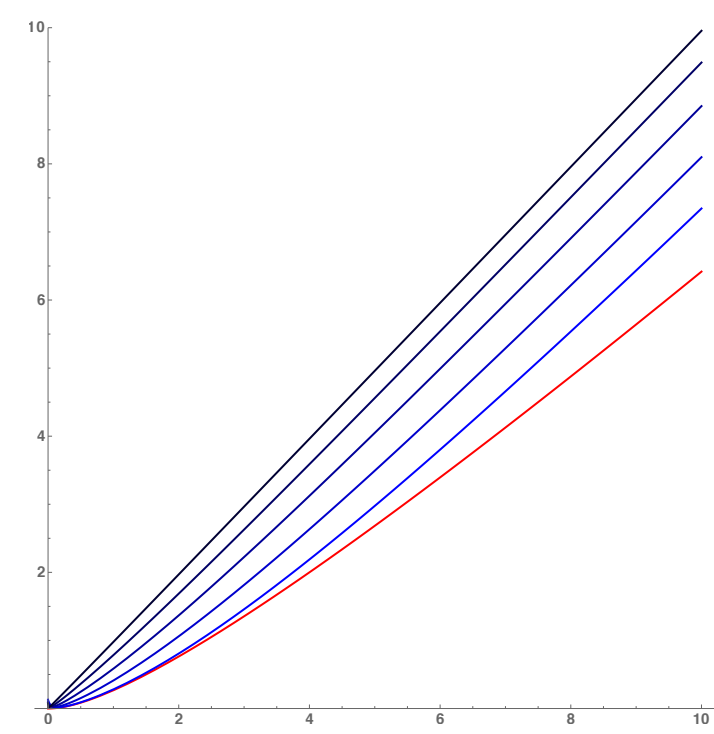

FiguRE 10. Graph of the right branch of $\mathcal{F}=0$ (red) and $R_{1}(x, y)$ (blues), for $x \in(0,10)$ and random values $t \in\{2.1,3,4,5,5.9\}$.

A range of $t$ corresponding to the values $2<s<3$ is $-2<t<2$. We have the following result, where the value of $s^{*}$ is given in Remark 6 .

Proposition 10. If $a=b=3$ and $2<s<s^{*}=2.99999 \ldots<3$, then the separatrix $\mathcal{S}$ of the saddle point $(\bar{x}, \bar{y})$ in the first quadrant of system (2) is bounded above by $h_{3}(x, y)=$ $y-(x+1-\sqrt{2 x+1})=0$, which is contained into $\mathcal{F}=0$ with $a=3$, and bounded below by the (4,3)-Padé approximation

$$
R_{2}=y-\frac{r_{0}(t)+r_{1}(t) x+r_{2}(t) x^{2}+r_{3}(t) x^{3}+r_{4}(t) x^{4}}{r_{4}(t)\left(3 x^{3}+10^{6} x^{2}+1\right)}=0,
$$

where $r_{i}(t)$ are certain polynomials of degree 17 in $t, i=0,1,2,3$, with rational coefficients and

$$
r_{4}(t)=(t-14)^{3}\left(t^{2}+92\right)^{4}\left(5 t^{2}-60 t-716\right)\left(t^{2}-12 t-124\right)^{2} .
$$


Proof. As in the previous result, we analyze the signs of $M_{R_{2}}$ and $M_{h_{3}}$. Again after Corollary 8 , we know that $h_{3}=0$ is above $\mathcal{S}$ for $2<s<3$. Indeed $M_{h_{3}}>0$. Next we check the sign of $M_{R_{2}}$. We have

$$
M_{R_{2}}=\frac{B(x, t)(x-\bar{x})^{4}}{r_{4}(t)^{2}\left(3 x^{3}+10^{6} x^{2}+1\right)^{3}},
$$

where $B(x, t)=\sum_{i=0}^{7} b_{i}(t) x^{i}$ is a polynomial of degree 7 in $x$. Here the $b_{i}(t)$ are polynomials in $t$ of large degree with rational coefficients that we do not write. Usual arguments prove that $b_{3}(t)>0$ and that $b_{i}(t)<0$, for $i=1,2,4,5,6,7,-2<t<2$. Moreover, the polynomial $b_{0}(t)$ has a simple root at some value $t=t^{\star} \approx-1.999988 \ldots$, so we restrict our study to $t^{\star}<t<2$, because $M_{R_{2}}$ is positive when $x$ is close enough to zero if $-2<t<t^{\star}$, and we want it to be always negative for all $x>0$. This value $t^{\star}$ is a zero of the polynomial

$$
\begin{aligned}
& -32470258907025358962688-1501056968874809016320 t+4527643659681763979264 t^{2} \\
& -1061485622793312296960 t^{3}+140023272828998468608 t^{4}-15576502915830445056 t^{5} \\
& +1590608240444014336 t^{6}-157044948711552000 t^{7}+17169730489332416 t^{8} \\
& -1734721741694272 t^{9}+126259806149072 t^{10}-5977555356768 t^{11}+174284624652 t^{12} \\
& -2847822604 t^{13}+19996445 t^{14}
\end{aligned}
$$

which is a factor of $b_{0}(t)$. The value of $s^{*}$ can be achieved from $t^{*}$.

We want to prove that $M_{R_{2}}<0$ for all $x>0, x \neq \bar{x}$, and for all $t \in\left(t^{*}, 2\right)$.. It is easy to check that its denominator is positive for $-2<t<2$ and $x>0$. We claim that $B(x, t)<0$ for all $x>0$ and $t^{\star}<t<2$.

To prove the claim we first note that $B(0, t)<0$ for $t \in\left(t^{\star}, 2\right)$ and that $\lim _{x \rightarrow \infty} B(x, t)=$ $-\infty$, since $b_{7}(t)<0$ for all $t \in(-2,2)$. Now we compute the discriminant $\mathcal{D}(t)$ of the polynomial $B(x, t)$ with respect to $x$, see [21]. This discriminant is a polynomial of degree 312 in $t$. It vanishes for some values of $t \in\left(t^{\star}, 2\right)$, but the number of positive zeros of $B(x, t)$ is always zero. This can be seen by using the method developed in [7, App. 2] that allows to check the desired property taking a single (rational) value of $t$, say $\bar{t}$, in each of the subintervals $\left(t^{\star}, 2\right)$ obtained after removing the zeroes of $\mathcal{D}$. It turns out that each of the polynomials $B(x, \bar{t})$ constructed with the described procedure does not change sign for $x>0$ (it has either one or three negative zeros and the rest of zeros are complex). Hence the claim follows, and therefore for $x>0, x \neq \bar{x}$, and $t \in\left(t^{*}, 2\right)$, we have proved that $M_{R_{2}}<0$.

Taking into account that both gradients of $h_{3}$ and $R_{2}$ point upwards, the proposition follows.

Figure 11 shows the algebraic bounds of $\mathcal{S}$ for some values of $t$. Note that when $t \rightarrow-2$ the upper bound tends to the $x$ axis. Indeed, $t=-2$ means $s=3$, for which the saddle in the first quadrant collapses to the node on the $x$ axis. Notice also that joining both Figures 10 and 11 we can see how our approximation of $\mathcal{S}$ moves from the straight line $y=x$ (for $s=1$ ) to the straight line $y=0$ (for $s=3$ ). Recall that $\mathcal{S}$ is algebraic for $1<s<3$ if and only if $s=2$, and in this case it coincides with $\mathcal{F}=0$.

Remark 6. The value $s^{*}$ in Proposition 10 comes from (7) applied to $t^{\star}$, which is given in the proof of this proposition. It is very close to 3 , which is the maximum value of s allowed, since $s<b=3$. Recall again that when $s=3$ the saddle collapses to the node $(1,0)$ located in the $x$-axis. The point is of saddle-node type when $s=3$.

To end this section, we plot the estimation of $\kappa(R)$ for $R=10$ and $t^{*}<t<6$, that is $1<s<s^{*}$, see Figure 12. We notice that $\kappa(10)$ lays, for all the values of $t$, between the red and the blue curves. Hence the estimations of $\kappa(10)$ for all possible values of $s$ assure that $\kappa(10)>1$. 


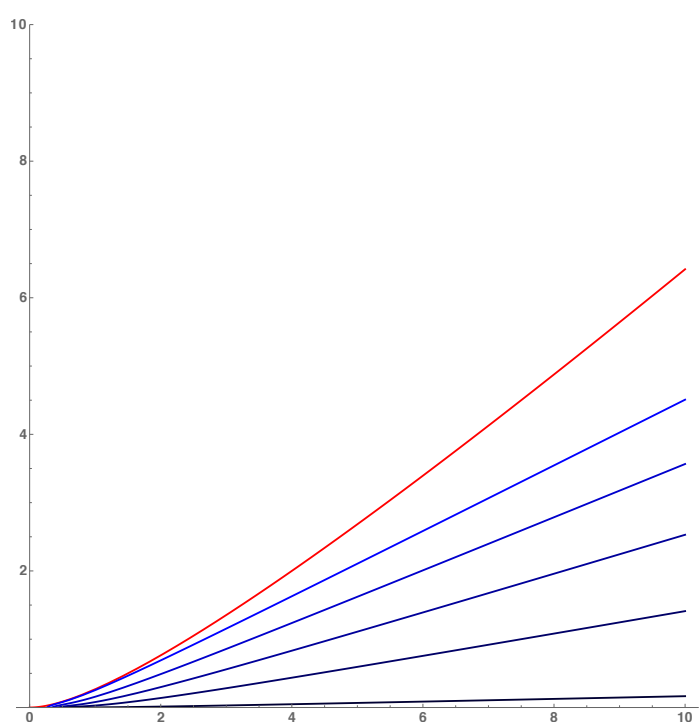

FigURE 11. Graph of the right branch of $\mathcal{F}=0$ (red) and $R_{2}(x, y)=0$ (blues), for $x \in(0,10)$ and random values $t \in\{1.9,1,0,-1,-1.9\}$.

The ratio of areas of $\mathcal{F}=0$ is constant and equal to

$$
\frac{40+\frac{1}{3}(21 \sqrt{21}-1)}{60+\frac{1}{3}(1-21 \sqrt{21})} \sim 2.539
$$

for all $t$ since $\mathcal{F}$ does not depend on $t$. Indeed this shows that $\kappa>1$ when $2<s<s^{*}$ without needing the lower bound $R_{2}=0$ of $\mathcal{S}$.

We also remark that for $t \rightarrow-2$, that is, $s \rightarrow 3, \kappa(R)$ takes large values. This is because in the case $s=3$ the saddle collapses to the node on the x axis, and hence the area below $\mathcal{S}$ tends to 0 and therefore $\kappa$ increases up to infinity as $R \rightarrow \infty$. On the other side, for $t \rightarrow 6$, that is, $s \rightarrow 1, \kappa(R)$ approaches the value 1 . This is because in the case $s=1 \mathcal{S}$ is the straight line $y=x$ (see Theorem 6 ), for which $A^{+}(R)=A^{-}(R)$, and so $\kappa(R) \rightarrow 1$ as $R \rightarrow \infty$.

Finally, note that there is a gap at $t=2$, that is $s=2$. This is because the algebraic approximation different from the curve $\mathcal{F}=0$ changes from upper bound to lower bound, so it changes from being above $\mathcal{F}=0$ to being below $\mathcal{F}=0$.

\section{ACKNOWLEDGEMENTS}

C. Chiralt, A. Ferragut and P. Vindel are partially supported by the Universitat Jaume I grant P1-1B2015-16. A. Ferragut and A. Gasull are partially supported by the MINECO grant MTM2013-40998-P. A. Gasull is partially supported by the Generalitat de Catalunya grant 2014SGR-568. P. Vindel is partially supported by the MINECO grant MTM201452016-C02-2-P.

\section{REFERENCES}

[1] A.A. Andronov, E.A. Leontovich, I.I. Gordon, A.G. Maier, Qualitative Theory of Second-Order Dynamic Systems, John Wiley \& Sons, New York 1973.

[2] Y.M. Bolaños Rivera, J. Llibre, C. Valls, Phase portraits of quadratic LotkaVolterra systems with a Darboux invariant in the Poincaré disc, Commun. Contemp. Math. 16 (2014), 1350041, 23 pp.

[3] L. Cairó, H. Giacomini, J. Llibre, Liouvillian first integrals for the planar LotkaVolterra system, Rend. Circ. Mat. Palermo 52 (2003), 389-418. 


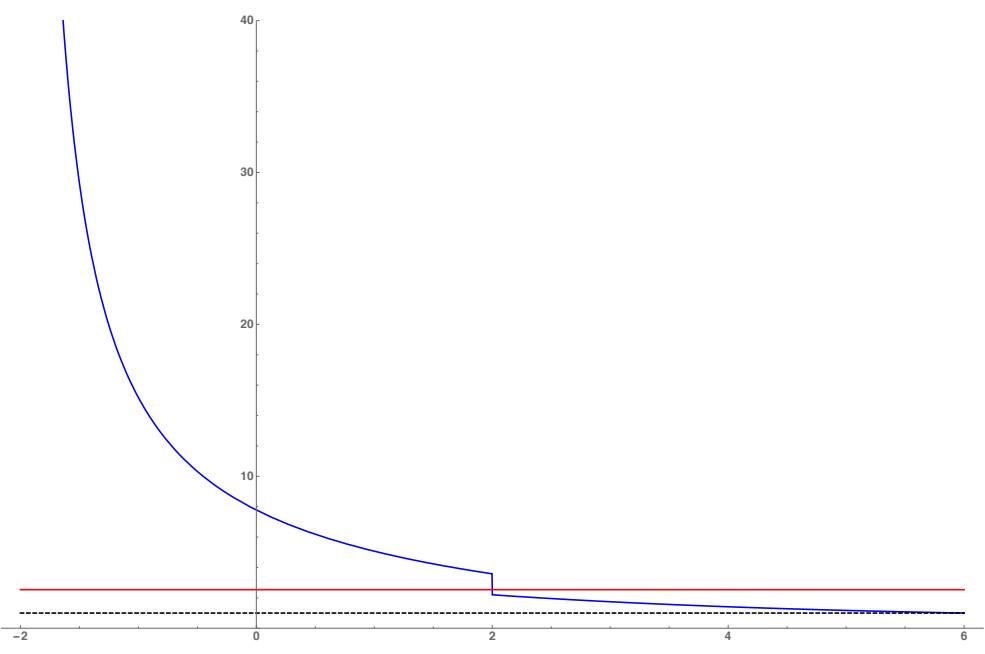

Figure 12. Graph of the range of $\kappa(10)$ in terms of $t \in\left\{t^{*}, 6\right\}$. The ratio of areas of $\mathcal{F}=0$, which is constant for all $t$ since $\mathcal{F}$ does not depend on $t$, is drawn in red; the ratio corresponding to the Padé approximations $R_{1}$ and $R_{2}$ is shown in blue. The black dashed straight line is the value 1 . The value $\kappa(10)$ lays, for all the values of $t$, between the red and the blue curves; therefore we have $\kappa>1$.

[4] J. Chavarriga, H. Giacomini, M. Grau, Necessary conditions for the existence of invariant algebraic curves for planar polynomial systems, Bull. Sci. math. 129 (2005), 99-126.

[5] A. Ferragut, J. Llibre, On the remarkable values of the rational first integrals of polynomial vector fields, J. Differential Equations 241 (2007), 399-417.

[6] I.A. García And M. GRAu, A survey on the inverse integrating factor, Qual. Theory Dyn. Syst. 9 (2010), 115-166.

[7] J. D. García-Saldaña, A. Gasull, H. Giacomini, Bifurcation values for a family of planar vector fields of degree five, Discrete Contin. Dyn. Syst. 35 (2015), 669-701.

[8] A. Gasull, H. Giacomini, S. Pérez-González, J. Torregrosa, A proof of Perko's conjectures for the Bogdanov-Takens system, J. Differential Equations 255 (2013), 2655-2671.

[9] A. Gasull, H. Giacomini, J. Torregrosa, Explicit upper and lower bounds for the traveling wave solutions of Fisher-Kolmogorov type equations, Discrete Contin. Dyn. Syst. 33 (2013), 3567-3582.

[10] G.F. Gause, The struggle for existence Baltimore, MD: Williams \& Wilkins, 1934.

[11] E.A.V. Gonzales, Generic properties of polynomial vector fields at infinity, Trans. Amer. Math. Soc. 143 (1969), 201-222.

[12] P.H. Leslie, An Analysis of the Data for Some Experiments Carried out by Gause with Populations of the Protozoa, Paramecium Aurelia and Paramecium Caudatum, Biometrika 44 (1957), 314-327.

[13] J. Llibre, Integrability of Polynomial Differential Systems, Handbook of Differential Equations, 437-532, Elsevier/North-Holland, Amsterdam 2004.

[14] J. Moulin-Ollagnier, Liouvillian Integration of the Lotka-Volterra system, Qual. T. Dyn. Sys. 2 (2001), 307-358. 
[15] J.D. Murray, Mathematical biology. Second edition. Biomathematics, 19. SpringerVerlag, Berlin, 1993.

[16] D. Schlomiuk, N. Vulpe, Global classification of the planar Lotka-Volterra differential systems according to their configurations of invariant straight lines, J. Fixed Point Theory Appl. 8 (2010), 177-245.

[17] D. Schlomiuk, N. Vulpe, Global topological classification of Lotka-Volterra quadratic differential systems, Electron. J. Differential Equations, No. 64, (2012), 69 pp.

[18] A. Seidenberg, Reduction of singularities of the differential equation $A d y=B d x$, Amer. J. Math. 90 (1968), 248-269.

[19] J. Sotomayor, Curvas Definidas por Equações Diferenciais no Plano, Institute de Matemática Pura e Aplicada, Rio de Janeiro, 1981.

[20] J. Stoer, R. Bulirsch, Introduction to numerical analysis. Translated from the German by R. Bartels, W. Gautschi and C. Witzgall. Springer-Verlag, New YorkHeidelberg, 1980.

[21] B. Sturmfels, Solving systems of polynomial equations. CBMS Regional Conference Series in Mathematics 97. Published for the Conference Board of the Mathematical Sciences, Washington, DC; by the American Mathematical Society, Providence, RI, 2002.

C. Chiralt, A. Ferragut, P. Vindel: Institut Universitari de Matemàtiques i Aplicacions de Castelló (imaC) and Departament de Matemàtiques, Universitat Jaume I, Edifici Ti (ESTEC), Av. de Vicent Sos Baynat, s/n, Campus del Riu Sec, 12071 Castelló de la Plana, Spain

E-mail address: chiralt@mat.uji.es, ferragut@uji.es, vindel@uji.es

A. Gasull: Departament de Matemàtiques, Universitat Autònoma de Barcelona, Edifici C, 08193 Bellaterra, Barcelona, Catalonia-Spain

E-mail address: gasull@mat.uab.cat 